\title{
UNIFORM ATTRACTORS FOR THE NON-AUTONOMOUS PARABOLIC EQUATION WITH NONLINEAR LAPLACIAN PRINCIPAL PART IN UNBOUNDED DOMAIN
}

\author{
GUANG-XIA CHEN
}

\begin{abstract}
In this paper, we are concerned with the asymptotic behavior of the solution for the non-autonomous parabolic equation with nonlinear Laplacian principal part in $\mathbb{R}^{n}$. The existence of the $\left(L^{2}\left(\mathbb{R}^{n}\right), L^{2}\left(\mathbb{R}^{n}\right)\right)$-uniform attractor, the $\left(L^{2}\left(\mathbb{R}^{n}\right), L^{p}\left(\mathbb{R}^{n}\right)\right)$-uniform attractor and the $\left(L^{2}\left(\mathbb{R}^{n}\right), W^{1, p}\left(\mathbb{R}^{n}\right) \cap L^{q}\left(\mathbb{R}^{n}\right)\right)$-uniform attractor will be proved.
\end{abstract}

Mathematics subject classification (2010): 35B40, 35B41, 35L70.

Keywords and phrases: uniform attractor, unbounded domain.

\section{REFERENCES}

[1] A. V. BABIN AND M. I. VishiK, Attractors of differential evolution equation in unbounded domain, Proc. Roy. Soc. Edinburgh Sect. A, 116 (1990), 221-243.

[2] A. V. BABin AND M. I. VishiK, Attractors of evolution equations, North-Holland, Amsterdam, 1992.

[3] A. RODRIGUEZ-BERNAL AND B. WANG, Attractors for partly dissipative reaction diffusion equation systems in $\mathbb{R}^{n}$, J. Math. Anal. Appl., 252 (2000), 790-803.

[4] V. V. Chepyzhov And M. I. VishiK, Attractors for Equation of Mathematical Physics, American Mathematical Society, Providence, Rhode Island, 2002.

[5] A. N. CARVAlho AND C. B. Gentile, Asymptotic behaviour of nonlinear parabolic equations with monotone principal part, J. Math. Anal. Appl., 280 (2003), 252-272.

[6] J. W. Cholewa And T. Dlotko, Global attractors in abstract parabolic problems, Cambridge University Press, 2000.

[7] D. N. Cheban, P. E. Kloeden, B. Schmalfuss, The relationship between pullback, forward and global attractors of non-autonomous dynamical systems, Nonlinear Dyn. Syst. Theory, 2 (2002), 9-28.

[8] E. Feireisl, Ph. Laurencot, F. Simondon, H. Toure, Compact attractors for reaction-diffusion equation in $\mathbb{R}^{n}$, C. R. Acad. Sci. Paris. Sér. I Math., 319 (1994), 147-151.

[9] E. Feireisl, Ph. Laurencot and F. Simondon, Compact attractor for degenerate parabolic equation in $\mathbb{R}^{n}$, C. R. Acad. Sci. Paris. Sér. I Math., 320 (1995), 1079-1083.

[10] E. Feireisl, Ph. LAURENCOT AND F. Simondon, Global attractors for degenerate parabolic equation on unbounded domains, J. Differential Equations, 129 (1996), 239-261.

[11] J. L. Lions, Quelques Méthodes de Résolution des Problèmes aux Limites Non Linéaires, Dunod, Paris, 1969.

[12] A. KH. Khanmanedov, Existence of a global attractor for the degenerate parabolic equation with p-Laplacian principal part in unbounded domain, J. Math. Anal. Appl., 316 (2006), 601-615.

[13] ChUn-YOu Sun AND CHENG-KUI ZHONG, Attractor for semilinear reaction-diffusion equation with distribution derivatives in unbounded demains, Nonlinear Anal., 63 (2005), 49-65.

[14] B.WANG, Attractors for reaction diffusion equation in unbounded domains, Phys. D, 128 (1999), $41-52$.

[15] Mei-Hua Yang, Chun-You Sun And Cheng-Kui Zhong, Global attractor for p-Laplacian equation, J. Math. Anal. Appl., 327 (2007), 1130-1142.

[16] Mei-Hua Yang, Chun-You Sun And Cheng-Kui Zhong, Existence of a global attractor for a p-Laplacian equation in $\mathbb{R}^{n}$, Nonlinear Anal., 66 (2007), 1-13. 
[17] Cheng-Kui Zhong, Mei-Hua Yang and Chun-You Sun, The existence of global attractors for the norm-to-weak continuous semigroup and application to the nonlinear reaction-diffusion equations, J. Differential Equations, 15 (2006), 367-399.

[18] GUANG-XIA CHEN AND CHENG-KUI ZHONG, Uniform attractor for non-autonomous p-Laplacian equation, Nonlinear Anal., 68 (2008), 3349-3363. 